\title{
Um Modelo Numérico Determinístico de Previsão no Contexto da Teoria do Controle Ótimo
}

P.S.D. DA SILVA ${ }^{1}$,Laboratório de Ciências Matemáticas, LCMAT/UENF, 28013602 Campos dos Goytacazes, RJ, Brasil.

P.H.G. OLIVEIRA ${ }^{2}$, G. MORALES ${ }^{3}$, Laboratório de Engenharia de Produção, LEPROD/UENF,28013-602 Campos dos Goytacazes, RJ, Brasil.

Resumo. Assimilação Variacional de Dados e Equações Adjuntas são dois métodos que, combinados, originam uma metodologia capaz de fornecer uma condição inicial de um sistema em estudo bastante realística e adequada a simulações numéricas, a um custo computacional aceitável e temporal que permite tomadas de decisão mais eficientes no caso de certos acidentes ambientais, quando apenas algumas observações e a dinâmica do sistema são conhecidas. Neste artigo, os fundamentos matemáticos e os procedimentos para se obter o adjunto de um dado programa computacional, uma tarefa fundamental para a aplicação da metodologia, são examinados.

Palavras-chave. Assimilação variacional de dados, Método das equações adjuntas.

\section{Introdução}

O estudo dos escoamentos geofísicos, sejam atmosféricos ou oceânicos, vêm revelando, desde a implementação operacional de um modelo numérico de previsão do tempo já na década de 1950, características que dificultam extremamente a simulação computacional desses fenômenos. Os modelos numéricos empregados nas simulações de escoamentos geofísicos são representações, na forma discreta, das leis físicas que os governam, em geral, um sistema evolutivo de equações diferenciais parciais [11]. Portanto, para a simulação numérica da evolução espaço-temporal de um escoamento em estudo, é necessário o conhecimento de sua condição inicial, isto é, a contrapartida numérica do estado ou configuração inicial real do escoamento em estudo.

Contudo, a distribuição de uma rede de observações eficiente nos domínios espaciais dos escoamentos geofísicos é sempre dificultada pela própria natureza (tamanho e localização) desses domínios, acarretando uma escassez de informações sobre o fenômeno em estudo capaz de comprometer a qualidade das simulações computacionais [14]. Além disso, esses escoamentos contêm aspectos de difícil representação

\footnotetext{
${ }^{1}$ paulosd@uenf.br.

${ }^{2}$ phgoliveira@ibest.com.br. A autora agradece o apoio da CAPES.

${ }^{3}$ gudelia@uenf.br.
} 
em um modelo numérico e mesmo em modelos analíticos, sendo ainda, em certas situações, processos extremamente sensíveis a variações em suas condições iniciais [9]. Portanto, estabelecer uma condição inicial adequada às simulações é, neste caso, decisivo para a qualidade dos resultados obtidos.

Entende-se por uma condição inicial adequada aos experimentos numéricos aquela que, ao ser empregada em uma simulação da dinâmica do sistema, gera estados do modelo numérico que reproduzam, com uma precisão dentro dos limites préestabelecidos, as observações disponíveis. Uma maneira de estabelecer-se a condição inicial adequada às simulações computacionais é, neste caso, considerar, além da dinâmica do escoamento, todo o conjunto de informações disponível por observação sobre o sistema em estudo. Isto é exatamente o que faz a metodologia conhecida pela comunidade que investiga escoamentos geofísicos como Assimilação de Dados [5].

Enquanto a modelagem do comportamento da mancha de óleo nas primeiras horas após o derrame [3] e os métodos numéricos para o tratamento computacional de equações envolvendo tanto termos advectivos quanto difusivos [1] têm sido extensamente abordados na literatura há um tempo considerável, o problema da determinação da condição inicial para simulações de escoamentos geofísicos, exceto no caso dos modelos numéricos de previsão do tempo, ainda não tem recebido a mesma atenção. Este último aspecto é o foco principal deste trabalho.

Um problema envolvendo escoamentos geofísicos, em sua forma discreta, produz um número muito elevado de variáveis (na faixa de $10^{5}-10^{7}$ ), de sorte que a Assimilação Variacional de Dados é, de imediato, uma metodologia computacionalmente intratável. Uma solução para esta dificuldade é obtida com a utilização do método das Equações Adjuntas, que adequadamente insere o problema da determinação de uma condição inicial para a simulação de escoamentos geofísicos no domínio da Teoria do Controle Ótimo [7], e que terá seus fundamentos matemáticos aqui analisados com o necessário rigor. Uma segunda conseqüência da metodologia que surge da combinação da Assimilação Variacional de Dados com o método das Equações Adjuntas é a transformação do problema de minimização com restrição, como originalmente proposto pelo formalismo da Assimilação Variacional de Dados, num outro de minimização sem restrições, possibilitando a utilização de rotinas mais eficientes, como a L-BFGS, na determinação de uma condição inicial ótima com o emprego dessa metodologia.

A eficiência dessa metodologia, comprovada por modelos numéricos de previsão do tempo já operacionais [10], tem produzido um formidável crescimento no leque de suas aplicações [2], [12], [4], o que requer uma contínua utilização de resultados teóricos cada vez mais complexos, o que, aliado à rapidez com que esses modelos surgem, dificulta o pleno entendimento de suas bases teóricas. Atenuar esse efeito, é outro dos objetivos deste trabalho.

Na seção seguinte, são apresentados os fundamentos matemáticos dos dois métodos cruciais a este trabalho: a Assimilação Variacional de Dados e o Método das Equações Adjuntas. Em seguida, descreve-se um experimento numérico bidimensional, apresentando-se com algum detalhamento a estrutura de um programa em FORTRAN, desenvolvido pelos autores, para realizar a Assimilação Variacional de Dados de um escoamento governado pela equação de advecção-difusão bidimen- 
sional, com o uso de sua equação adjunta, indicando os processos matemáticos realizados por cada sub-rotina do programa.

\section{Formulação Matemática da Metodologia}

A metodologia para a geração de uma condição inicial ótima para simulações computacionais de problemas de grande escala apresentada neste trabalho tem por base a Assimilação Variacional de Dados e o Método das Equações Adjuntas. Trata-se, a seguir, da formulação matemática dos métodos que serão empregados diretamente no desenvolvimento do algoritmo para a execução da metodologia.

\subsection{O método da Assimilação Variacional de Dados}

Seja $S$ um sistema evolutivo, observado durante o intervalo de tempo $\left[t_{1}, t_{2}\right]$, do qual se dispõe de um conjunto de observações, coletadas no mesmo intervalo de tempo e distribuídas no domínio espacial onde reside o foco de interesse em $S$. Além disso, é conhecida a dinâmica de $S$. Com base nessas informações, quer-se determinar a condição inicial do modelo de $S$, ou seja, a representação numérica da configuração de $S$ no instante de tempo $t_{1}$ a partir da qual uma simulação computacional de $S$ gere configurações que se aproximem, dentro de uma precisão pré-estabelecida, das observações disponíveis de $S$ em instantes de tempo correspondentes, com o objetivo de se obter uma configuração do modelo em $t_{f}>t_{2}$ que venha a reproduzir a real configuração de $S$ no instante $t_{f}$ também dentro da precisão aceita. Este processo, conhecido como Assimilação de Dados é, conceitualmente, semelhante à Melhor Estimativa Linear Não Tendenciosa [13] da configuração de $S$ em $t_{f}$, dado um conjunto de configurações de $S$ em $\left[t_{1}, t_{2}\right]$, sendo aqui resolvido, numa abordagem determinística, como um problema de controle ótimo, no qual a condição inicial do sistema é o dado de controle.

Considera-se, inicialmente, a versão contínua do problema da Assimilação Variacional de Dados, por permitir um tratamento matemático já consagrado. Neste caso, os objetos matemáticos são:

1. as observações do sistema $S, Z:\left[t_{1}, t_{2}\right] \times G \rightarrow V$, tal que, $\forall t \in\left[t_{1}, t_{2}\right]$, $Z_{t}: G \rightarrow V$ é um operador diferenciável definido no subespaço vetorial $G$ contido no espaço vetorial de dimensão finita $V \operatorname{com}$ o produto interno $\langle\cdot, \cdot\rangle$, sendo $\left[t_{1}, t_{2}\right]$ o intervalo de assimilação.

2. a dinâmica do sistema $S$, dada por

$$
\frac{\partial X}{\partial t}=F(X)
$$

sendo $X:\left[t_{1}, t_{2}\right] \times G \rightarrow V$ a trajetória do sistema $S$ durante o intervalo de assimilação, $E=\left\{Y ; Y:\left[t_{1}, t_{2}\right] \times G \rightarrow V, Y 2\right.$ vezes diferenciável $\}$ e $F: E \rightarrow E$ um operador diferenciável.

3. a função "peso" $W:\left[t_{1}, t_{2}\right] \rightarrow L(G, V)$, sendo $L(G, V)$ o espaço vetorial dos operadores lineares em $V$ restritos ao subespaço $G$, que resulta das informações 
estatísticas dos instrumentos utilizados na coleta de informações sobre $S$ e que, a cada $t \in\left[t_{1}, t_{2}\right]$, associa o operador linear injetivo $W(t): G \rightarrow V$.

4. o funcional quadrático

$$
\begin{aligned}
J: E & \rightarrow \mathbb{R} \\
X & \mapsto \frac{1}{2} \int_{D}\langle W(t)(X(t, \vec{x})-Z(t, \vec{x})), W(t)(X(t, \vec{x})-Z(t, \vec{x}))\rangle d D,
\end{aligned}
$$

sendo $D=\left\{(t, \vec{x}) ; t \in\left[t_{1}, t_{2}\right], \vec{x} \in G\right\}$.

O problema da Assimilação Variacional de Dados é, então, encontrar a trajetória dos estados de $S, X:\left[t_{1}, t_{2}\right] \times G \rightarrow V$ tal que $X$ seja a solução de (2.1) que minimiza o funcional (2.2), ou seja, é equivalente ao seguinte problema de minimização com restrição:

Problema $M_{R}$ : encontrar a solução de 2.1 que minimiza 2.2

Como a solução do problema da Assimilação de Dados será obtida numericamente, é necessário resolver sua versão discreta, o que, no entanto, acarreta uma outra dificuldade: o número de variáveis resultantes da versão discreta do conjunto de equações modelando a dinâmica de $S$ bem como seu domínio espaço-temporal é extremamente grande, como visto na Introdução. Portanto, computacionalmente seria muito econômico (e, em muitos problemas, só assim exequível) se, em lugar da solução $X \in E$, se procurasse a solução $X_{1} \in E_{1}$, sendo $E_{1}=\left\{X_{1} ; X_{1}=\right.$ $\left.\left.X\right|_{\left\{t_{1}\right\} \times G}, X \in E\right\}$, a que se pode chamar o espaço das configurações iniciais de $S$, já que $X_{t_{1}}(G)=G$, que minimizasse a restrição do funcional $J$ a $E_{1}$.

A dificuldade aqui é não existir uma relação explícita entre $J$ e $X_{1}$, pois $J$ é uma função de $X$. No entanto, se o problema (2.1) for bem posto, no sentido de Hadamard, então o conhecimento de $X$ e o de $X_{1}$ são equivalentes. E o método das Equações Adjuntas fornece, partindo-se da relação entre $X$ e $X_{1}$, a diferencial da restrição de $J$ a $E_{1}$.

\subsection{O Método das Equações Adjuntas}

Reescreve-se o funcional (3) como

$$
\begin{aligned}
J: E & \rightarrow \mathbb{R} \\
X & \mapsto \int_{D} T(X(t, \vec{x})) d D,
\end{aligned}
$$

sendo

$$
\begin{aligned}
T: E & \rightarrow \mathbb{R} \\
X(\cdot, \cdot) & \mapsto \frac{1}{2}\langle W(\cdot)(X(\cdot, \cdot)-Z(\cdot, \cdot)), W(\cdot)(X(\cdot, \cdot)-Z(\cdot, \cdot))\rangle .
\end{aligned}
$$

Como a diferencial de $J$ em $X \in E$ é um funcional linear no espaço de Hilbert $E$ com o produto interno: 


$$
\langle X(\cdot, \cdot), Y(\cdot, \cdot)\rangle_{E}=\int_{D}\langle W(\cdot) X(\cdot, \cdot), W(\cdot) Y(\cdot, \cdot)\rangle d D
$$

obtém-se, $\forall H \in E$,

$$
d J(X) \cdot H=\left\langle\nabla_{X} J(X), H\right\rangle_{E}=\int_{D}\left\langle\nabla_{X} T(X(t, \vec{x})), H(t)\right\rangle d D,
$$

sendo $\nabla_{X} J$ e $\nabla_{X} T$, respectivamente, o gradiente de $J$ e o de $T$.

Considera-se, a seguir, a versão linear da equação 2.1:

$$
\frac{\partial X}{\partial t}-\frac{\partial F}{\partial x} X=0
$$

que resulta da substituição do operador $F$ pela sua aproximação de primeira ordem em torno de um ponto de equilíbrio (omitido na equação) e na qual empregou-se a notação $\left(\frac{\partial F}{\partial x}\right)$ para sua diferencial nesse ponto. Considera-se, ainda, a seguinte equação adjunta não-homogênea da equação 2.5 :

$$
\frac{\partial X}{\partial t}+\left(\frac{\partial F}{\partial x}\right)^{*}+\nabla_{X} T=0
$$

sendo $\left(\frac{\partial F}{\partial x}\right)^{*}$ o adjunto do operador $\left(\frac{\partial F}{\partial x}\right)$. Tem-se, então, o seguinte resultado:

Teorema 2.1. Dada uma solução $X \in E$ de $M_{R}, X_{1} \in E_{1}, X_{1}=\left.X\right|_{\left\{t_{1}\right\} \times G}$, é solução do seguinte problema de minimiação sem restrição:

Problema $M_{S}$ : encontrar $X_{1} \in E_{1}$ que minimiza $\left\langle\nabla_{X_{1}} J, X_{1}\right\rangle$.

Demonstração. Sejam $Y:\left[t_{1}, t_{2}\right] \times G \rightarrow V$ a solução de 2.6 tal que $Y_{t_{2}}=0$ e $X \in E$ uma solução qualquer de 2.5 , tem-se então que

$$
\left\langle\frac{\partial X}{\partial t}-\frac{\partial F}{\partial x} X, Y\right\rangle_{E}+\left\langle\frac{\partial Y}{\partial t}+\left(\frac{\partial F}{\partial x}\right)^{*} Y+\nabla_{X} T,\right\rangle_{E}=0
$$

Donde

$$
\frac{\partial}{\partial t}\langle Y, X\rangle_{E}=-\left\langle\nabla_{X} T, X\right\rangle_{E}=0
$$

E, integrando, vem

$$
\left.\langle Y, X\rangle_{E}\right|_{t_{1}} ^{t_{2}}=-\int_{t_{1}}^{t_{2}}\left\langle\nabla_{X} T, X\right\rangle_{E} d t=-\left\langle\nabla_{X} J, X\right\rangle_{E}
$$

que, com as condições iniciais do problema, fornece

$$
\left\langle Y_{1}, X_{1}\right\rangle_{E_{1}}=\left\langle\nabla_{X} J, X\right\rangle_{E}
$$


O significado de 2.7 deve ficar claro: seu lado direito é a expressão da diferencial de $J$ (em relação a $X), d J(X)$. Como $X_{1}$ é a projeção de $X$ sobre $E_{1}$, o lado esquerdo de 2.7 é a expressão da diferencial da restrição de $J$ (em relação a $X_{1}$ ) a $E_{1}$. Pela unicidade da representação da última diferencial como um produto interno em $E_{1}$, tem-se que $Y_{1}$ é precisamente o gradiente da restrição do funcional $J$ a $E_{1}$, ou seja, $Y_{1}$ é a projeção de $\nabla_{X} J$ sobre $E_{1}$, isto é,

$$
Y_{1}=\nabla_{X_{1}} J
$$

Portanto, com o emprego do Método das Equações Adjuntas, projeta-se o conjunto $C^{2}\left(\left[t_{1}, t_{2}\right] \times V, V\right)$ sobre $C^{2}\left(\left\{t_{1}\right\} \times V, V\right)$, o que representa, do ponto de vista computacional, uma redução considerável no número de variáveis do problema discreto. Além disso, essa projeção transforma um problema de minimização com restrição num problema de minimização sem restrição, o que permite o emprego de rotinas de minimização para problemas de grande porte sem restrições, um procedimento que também reduz o esforço computacional total.

O problema $M_{R}$, envolvendo o funcional quadrático $J$ definido em 2.2 tem a existência de sua solução garantida, mas não a sua unicidade. Para obter a unicidade da solução de $M_{R}$, e de $M_{S}$, redefine-se o problema para o seguinte funcional quadrático:

$$
\begin{aligned}
J_{T}: E & \rightarrow \mathbb{R} \\
X & \mapsto J_{b}(X)+J(X),
\end{aligned}
$$

sendo o funcional $J_{b}$ definido como

$$
\begin{aligned}
J_{b}: X & \rightarrow \mathbb{R} \\
X & \left.\mapsto \frac{1}{2} \int_{G}\left\langle B\left(X_{1}-X_{b}\right)\right), B\left(X_{1}-X_{b}\right)\right\rangle_{E_{1}} d x
\end{aligned}
$$

onde $X_{1}=X_{t_{1}}, X_{b}$ é uma estimativa disponível da configuração inicial de $X$ e $B \in L(G, V)$ é o operador determinado pelas informações estatísticas disponíveis sobre a estimativa $X_{b}$. Tem-se, então, o resultado a seguir.

Teorema 2.2. Dado o problema:

Problema $M_{R^{\prime}}$ : encontrar a solução de 2.1 que minimiza 2.9,

existe uma única solução $X$ de $M_{R^{\prime}}$, além disso $X_{1}=\left.X\right|_{E_{1}} \in E_{1}$ é a solução do problema

$$
M_{S^{\prime}} \text { : encontrar } X_{1} \in E_{1} \text { que minimiza }\left\langle\nabla_{X_{1}} J_{T}, X\right\rangle_{E_{1}}
$$

Demonstração. Basta definir o produto interno em $E$ como

$$
\begin{gathered}
\langle X(\cdot, \cdot), Y(\cdot, \cdot)\rangle_{E}=\int_{D_{1}}\left\langle B\left(X\left(t_{1}, \cdot\right)\right), B\left(Y\left(t_{1}, \cdot\right)\right)\right\rangle d D_{1}+ \\
+\int_{D}\langle W(\cdot)(X(\cdot, \cdot)), W(\cdot)(Y(\cdot, \cdot))\rangle d D
\end{gathered}
$$

e utilizar o desenvolvimento que precede o 2.1 e a argumentação de sua demonstração. 
Observe-se que, conhecida a estimativa $X_{b}$ da trajetória no instante $t_{1}$, a solução de $M_{S^{\prime}}$ é da forma $X_{1}=X_{b}+h$, ou seja, a solução $X_{1}$ de $M_{S^{\prime}}$ é obtida, iterativamente, por "perturbações" de $X_{b}$ até conseguir-se aquela cuja trajetória resultante minimiza $M_{R^{\prime}}$.

A versão discreta da Assimilação Variacional de Dados e do método da Equação Adjunta é derivado com facilidade da versão contínua: dada o conjunto discreto de instantes de tempo, $U=\left\{\tau_{1}, \tau_{2}, \ldots, \tau_{k}\right\}$, no intervalo de assimilação $\left[t_{1}, t_{2}\right]$ para o qual há observações de $S$, definem-se $Z: U \times G \rightarrow V, X: U \times G \rightarrow V, W: U \rightarrow$ $L(G, V)$ e o funcional 2.2 como o somatório:

$$
\begin{aligned}
J_{D} & : E_{D} \rightarrow \mathbb{R} \\
X & \mapsto \frac{1}{2} \sum_{i}\left\langle W\left(\tau_{i}\right)\left(X\left(\tau_{i}, \vec{x}\right)-Z\left(\tau_{i}, \vec{x}\right)\right), W\left(\tau_{i}\right)\left(X\left(\tau_{i}, \vec{x}\right)-Z\left(\tau_{i}, \vec{x}\right)\right)\right\rangle,
\end{aligned}
$$

sendo $E_{D}=\{X ; X: U \times G \rightarrow V\}$.

Com essas considerações, pode-se apresentar o resultado de um experimento numérico.

\section{Experimento Numérico}

Estudou-se o problema da determinação ou da predição determinística da evolução de uma mancha produzida por um derrame de óleo em uma região do mar cuja hidrodinâmica é conhecida, dispondo-se também de um conjunto de observações da mancha no intervalo de tempo $\left[t_{1}, t_{2}\right]$. As observações são todas incompletas, de modo que, em qualquer instante $t_{1} \leq t \leq t_{2}$, a configuração disponível, $Z(t)$, não reproduzindo integralmente a mancha de óleo, não poderia ser utilizada como "condição inicial" para a integração do modelo numérico.

$\mathrm{Na}$ figura 1 apresenta-se o fluxograma do programa para a Assimilação Variacional de Dados combinado ao Método da Equação Adjunta.

Simulou-se um derrame de óleo em um domínio espacial de $6000 \mathrm{~m} \times 4400 \mathrm{~m}$, discretizado por uma malha bidimensional de $21 \times 21$ nós, sendo $\Delta x=300 \mathrm{~m}, \Delta y=$ $220 \mathrm{~m}$ e o intervalo de tempo para a análise [0,30000] em segundos, com $\Delta t=300 \mathrm{~s}$. Tomou-se a velocidade da superfície do mar como $\vec{u}=(u, v)$, sendo $u=1.10^{-2} \mathrm{~m} \times$ $s^{-1}$ e $v=0$., $D$ o coeficiente de difusividade variável e $C$ a concentração do óleo. O transporte do óleo foi descrito pela equação de advecção-difusão bidimensional:

$$
\frac{\partial C}{\partial t}+u \frac{\partial C}{\partial x}+v \frac{\partial C}{\partial y}-D\left(\frac{\partial^{2} C}{\partial^{2}}+\frac{\partial^{2} C}{\partial^{2} y}\right)=0
$$

que é o modelo de transporte de óleo na superfície do mar universalmente empregado, quando se supõe o óleo sujeito somente à ação da água [6].

Considerando, então, como condição inicial para a simulação numérica a mancha de óleo produzida logo após cessar a ação da gravidade (figura 2), integrou-se 3.1 (em $G(U)$ ) e foram armazenadas, como observações do sistema, as configurações obtidas nos instantes $t=10 \times i \times \Delta t, i=1, \ldots 9$. Nas figuras 3 e 4 , são mostradas as configurações parciais da mancha de óleo para $t=20 \times \Delta t$ e $t=90 \times \Delta t$. Perturbouse, então, a configuração inicial aleatoriamente, utilizando-se a função RAND da 


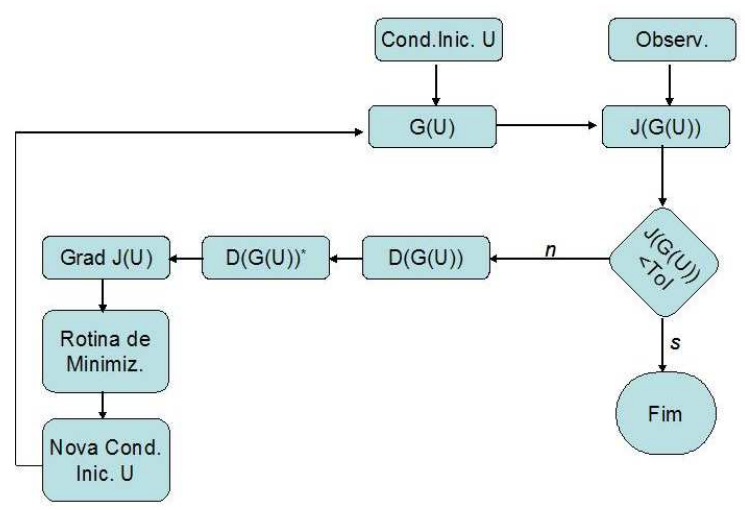

Figura 1: Fluxograma para Assimilação Variacional de Dados com o Método das Equações Adjuntas.

biblioteca do Fortran 90, obtendo-se a configuração inicial perturbada (figura 5). A partir de então tem início o processo de Assimilação Variacional de Dados: em $\mathrm{G}(\mathrm{U})$, a equação 3.1 é integrada com a condição inicial perturbada e, se o erro entre as configurações geradas pelo modelo e as observações (avaliado em $J(G(U))$ ) estiver acima da tolerância estabelecida, o programa principal chama a sub-rotina $D(G(U))^{*}$ para realizar a integração para trás no tempo da equação adjunta 2.6, gerando o gradiente do funcional $J$ com relação às condições iniciais do problema, que, passado à rotina de minimização L-BFGS [8], produz uma nova configuração inicial $U$. Essa nova condição inicial, ao ser integrada em $G(U)$, produzirá configurações do modelo numérico que diminuirão o valor de $J(G(U))$, até que este se situe abaixo da tolerância. Quando isso ocorrer, o modelo terá gerado a configuração inicial ótima (figura 6).

No experimento considerado, como as configurações geradas pelo modelo computacional foram tomadas como as observações do sistema (experimento dos "gêmeos idênticos"), a condição inicial ótima obtida pelo modelo numérico apresenta um erro menor que 1,5\% em relação à condição inicial do problema (figura 2).

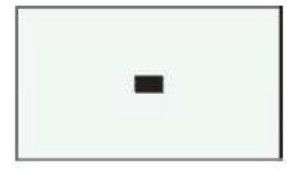

Figura 2: Configuração inicial do sistema.

Assim, se a condição inicial obtida pela Assimilação de Dados produziu configurações que, durante o intervalo de tempo $\left[t_{1}, t_{2}\right]$, melhor se aproximam das observações (no sentido examinado), para instantes $t_{f} \geq t_{2}$, a configuração $X_{t_{f}}$ é a melhor aproximação da real configuração do sistema no instante $t_{f}$ (no mesmo 


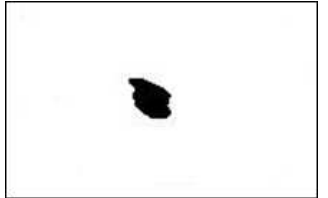

Figura 3: Configuração do sistema em $t=20 \times \Delta t$.

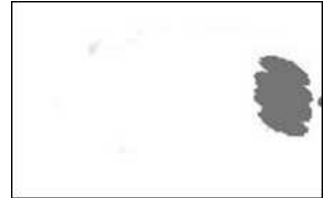

Figura 4: Configuração do sistema em $t=90 \times \Delta t$.

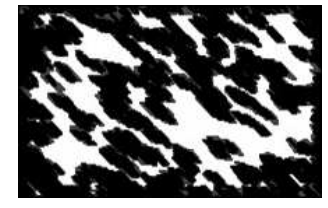

Figura 5: Configuração inicial perturbada.

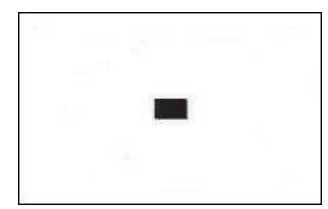

Figura 6: Configuração inicial recuperada.

sentido). Ou seja, as observações do sistema, no processo de Assimilação de Dados, atuam também como restrições à trajetória ótima $X \in E$, sendo então $X_{t_{f}}$ a melhor predição da configuração da mancha de óleo no instante $t_{f}>t_{2}$, dadas as observações $Z(t)$, com $t \in\left[t_{1}, t_{2}\right]$.

\section{Conclusões}

A metodologia que combina a Assimilação Variacional de Dados com o método da Equação Adjunta fornece, com um esforço computacional bastante reduzido, uma previsão confiável da trajetória de uma mancha de óleo à deriva no mar, previsão que, dispondo-se de uma rede de instrumentos para coleta de dados em áreas sob ameaça de vazamentos, permite traçar planos a fim de minimizar os efeitos nocivos do derrame. Por outro lado, novas questões teóricas vêm surgindo para a melhor aplicação da metodologia; uma delas, a das Observações Adaptativas, diz respeito justamente à disposição ótima de instrumentos de coleta de dados, que pode ainda ser investigada com um formalismo semelhante ao da Assimilação Variacional de Dados. 


\section{Referências}

[1] A.N. Brooks, T.J.R. Hughes, Streamline upwind Petrov-Galerkin formulations for convection dominated flows with particular emphasis on compressible Navier-Stokes equations, Comput. Methods Appl. Mech. Engrg., 32, No. 3 (1982), 199-259.

[2] H. Elbern, A. Strunk, H. Schimdt, O. Talagrand, Emission rate and chemical state estimation by 4-dimensional variational inversion, Atmos. Chem. Phys. 7 (2007), 3749-3769.

[3] J.A. Fay, Physical processes in the spread of oil on a water surface, In "Proceedings of the Joint Conference on Prevention and Control of Oil Spills", American Petroleum Institute, (1971), 463-467.

[4] M.I. Kamien, N.L. Schwartz, "Dynammic Optimization: The Calculus of Variation and Optimal Control in Economics and Management", North-Holland, 1981.

[5] F. X. LeDimet, O. Talagrand, Variational assimilation of meteorological observations with the adjoint equation I: Theory, Quart. J. Roy. Met. Soc., 113 (1997), 1311-1328.

[6] W.J. Lehr, H. M. Cekirge, Oil slick movements in the Arabian Golf, Proceeds of Petroleum and Marine Environment, Monaco, 1980.

[7] J.L. Lions, "Optimal Control of Systems Governed by Partial Differential Equations", Springer - Verlag, 1971.

[8] D. Liu, J. Nocedal, On the limited memory BFGS method for large scale minimization, Mathematical Programming, 45 (1989), 503-528.

[9] E.N. Lorenz, Deterministic non-periodic flows, Journal of Atmospheric Sciences, 20 (1963), 130-141.

[10] D.F. Parrish and J.D. Derber, The national meteorological center spectral statistical interpolation analysis system, Mon. Wea. Rev., 120 (1992), 1747--1763.

[11] J. Pedlosky, "Geophysical Fluid Dynamics", Springer, 1992.

[12] J. Reuther, A. Jameson, J. Farmer, L. Martinelli, D. Saunders, Aerodynamic shape optimization of complex aircraft configurations via an adjoint formulation, $A I A A,(1996)$, 96-0094.

[13] H.W. Sorensen, "Parameter Estimation: Principles and Problems", Marcel Dekker, 1980.

[14] O. Talagrand, Assimilation of observations, an introduction, Journal of Meteorological Society of Japan, 75, No. 1B (1997), 191-209. 